\title{
Comprehensive analysis of microRNA-regulated protein interaction network reveals the tumor suppressive role of microRNA-149 in human hepatocellular carcinoma via targeting AKT-mTOR pathway
}

Yanqiong Zhang ${ }^{1+}$, Xiaodong Guo ${ }^{2+}$, Lu Xiong ${ }^{3}$, Lingxiang Yu², Zhiwei Li ${ }^{2}$, Qiuyan Guo ${ }^{1}$, Zhiyan $\mathrm{Li}^{2}$, Boan $\mathrm{Li}^{2^{*}}$ and $\mathrm{Na} \operatorname{Lin}^{1 *}$

\begin{abstract}
Background: Our previous study identified AKT1, AKT2 and AKT3 as unfavorable prognostic factors for patients with hepatocellular carcinoma (HCC). However, limited data are available on their exact mechanisms in HCC. Since microRNAs (miRNAs) are implicated in various human cancers including HCC, we aimed to screen miRNAs targeting AKTs and investigate their underlying mechanisms in HCC by integrating bioinformatics prediction, network analysis, functional assay and clinical validation.

Methods: Five online programs of miRNA target prediction and RNAhybrid which calculate the minimum free energy (MFE) of the duplex miRNA:mRNA were used to screen optimized miRNA-AKT interactions. Then, miRNA-regulated protein interaction network was constructed and 5 topological features ('Degree', 'Node-betweenness', 'Edge-betweenness', 'Closeness' and 'Modularity') were analyzed to link candidate miRNA-AKT interactions to oncogenesis and cancer hallmarks. Further systematic experiments were performed to validate the prediction results.

Results: Six optimized miRNA-AKT interactions (miR-149-AKT1, miR-302d-AKT1, miR-184-AKT2, miR-708-AKT2, miR-122-AKT3 and miR-124-AKT3) were obtained by combining the miRNA target prediction and MFE calculation. Then, 103 validated targets for the 6 candidate miRNAs were collected from miRTarBase. According to the enrichment analysis on GO items and KEGG pathways, these validated targets were significantly enriched in many known oncogenic pathways for HCC. In addition, miRNA-regulated protein interaction network were divided into 5 functional modules. Importantly, AKT1 and its interaction with mTOR respectively had the highest node-betweenness and edge-betweenness, implying their bottleneck roles in the network. Further experiments confirmed that miRNA-149 directly targeted AKT1 in HCC by a miRNA luciferase reporter approach. Then, re-expression of miR-149 significantly inhibited HCC cell proliferation and tumorigenicity by regulating AKT1/mTOR pathway. Notably, miR-149 down-regulation in clinical HCC tissues was correlated with tumor aggressiveness and poor prognosis of patients.

(Continued on next page)
\end{abstract}

\footnotetext{
*Correspondence: boanli302@163.com; linna888@163.com

${ }^{\dagger}$ Equal contributors

${ }^{2} 302$ Hospital of PLA, Beijing 100039, China

'Institute of Chinese Materia Medica, China Academy of Chinese Medical

Sciences, Beijing 100700, China

Full list of author information is available at the end of the article
}

\section{Biomed Central}

(c) 2014 Zhang et al.; licensee BioMed Central Ltd. This is an Open Access article distributed under the terms of the Creative Commons Attribution License (http://creativecommons.org/licenses/by/4.0), which permits unrestricted use, distribution, and reproduction in any medium, provided the original work is properly credited. The Creative Commons Public Domain Dedication waiver (http://creativecommons.org/publicdomain/zero/1.0/) applies to the data made available in this article, unless otherwise stated. 


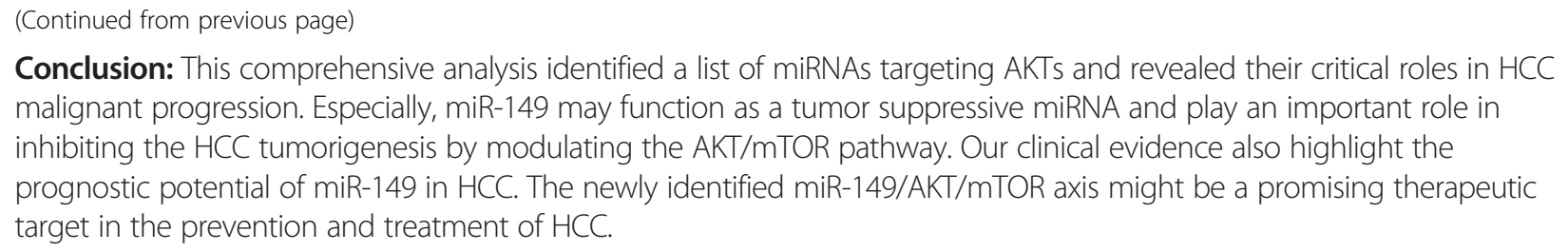

Keywords: Hepatocellular carcinoma, microRNA-regulated protein interaction network, microRNA-149, AKT/mTOR pathway, Prognosis

\section{Background}

Hepatocellular carcinoma (HCC) ranks as the fifth (seventh) most prevalent malignancy and the second (sixth) leading cause of cancer-related deaths in men (women), accounting for approximately 695,900 deaths per year worldwide [1]. The hepatocarcinogenesis is a multi-step process from chronic hepatitis, cirrhosis and dysplastic nodules to malignant tumors [2]. Despite the great advancement of numerous therapeutic strategies, HCC remains a major public health concern due to fast infiltrating growth, early metastasis, high-grade malignancy, and poor prognosis. More than two-thirds of HCC patients occur recurrence after surgical hepatic resection [3]. The overall 5-year survival rate for these patients is still only 5\% [4]. Growing clinical observations indicate that HCC patients with the same clinicopathologic features often display different outcome, suggesting that there may be several complex molecular and cellular events involved in the development and aggressive progression of HCC. Therefore, it is of crucial significance to investigate the molecular pathogenesis of HCC in order to develop novel therapeutic strategies for the treatment of this disease.

MicroRNAs (miRNAs) represent a group of short noncoding RNA molecules with 18-25 nucleotides in length [5]. Based on miRBase (release 21), the human genome encodes 1881 (2588) precursor (mature) miRNAs, which potentially target the majority of the human genes [6]. Functionally, miRNAs transcriptionally or post-transcriptionally suppress the expression of their target genes at mRNA or protein levels in many organisms, such as yeast, fruit flies, worms, vertebrates, human and plants by imperfect basepairing with the 3'untranslated regions (3'UTRs) of target genes [7], and play important roles in regulating diverse biological processes, including development, cell cycle, proliferation, differentiation, apoptosis and response to stress [8]. In recent years, accumulating evidence have also indicated that the dysregulation of miRNAs in various human cancers may modulate tumor cell proliferation, tumor angiogenesis, invasion and metastasis during tumor initiation and progression [9]. According to the functions of their target genes, miRNAs either act as oncomiRs or as tumor suppressors [10]. Since a growing number of HCC-related genes have been demonstrated to be regulated by miRNAs, it is not surprising that changes in the endogenous expression of miRNAs may be one of the most crucial mechanisms in the hepatocarcinogenesis. For example, miR-105 has been reported to function as a potential tumor suppressor and inhibit cell proliferation by regulating the PI3K/AKT signaling pathway in human HCC [11]; Tan and colleagues identified a serum of miRNA panel (hsa-miR-206, hsa-miR-141-3p, hsa-miR-433-3p, hsa-miR-1228-5p, hsa-miR-199a-5p, hsamiR-122-5p, hsa-miR-192-5p, and hsa-miR-26a-5p) that has considerable clinical value in HCC diagnosis [12]; The presence of miR-101 has also been indicated to be a biochemical marker for monitoring the progression of tumor development in HBV-related HCC, and to be a potential prognostic marker and therapeutic target for HCC [13]. These previous findings highlight the central and potential roles of miRNAs in the pathogenesis of HCC and elucidate new possibilities that may be useful as diagnostic and prognostic markers, as well as novel therapeutic targets in HCC. However, the existing knowledge of miRNA alterations in human HCC remains limited.

MiRNAs have been demonstrated to function in a multiple-to-multiple relationship with their target genes, which means that a specific miRNA can regulate expression of up to thousand mRNAs, and a specific mRNA can be regulated by multiple miRNAs, implying that the interference of miRNAs in biological processes is quite complicated [14]. Since miRNAs and their target mRNAs function cooperatively, we should construct the miRNAs regulatory networks in order to scrutinize the effects of miRNAs by the network-based systems biology approaches. Our previous study combined expression profile, interaction network analysis and clinical validation to identify three AKT kinase family members (AKT1 AKT3) as unfavorable prognostic factors for HCC patients [15]. However, limited data are available on their exact mechanisms in this cancer. To investigate this issue, we here performed a comprehensive analysis by integrating bioinformatics prediction of miRNAs targeting AKTs, miRNAs-regulated protein interaction network construction and topological analysis, and extensive validation on the miRNA-AKT functional 
interaction and their clinical relevance in human HCC as shown in Figure 1.

\section{Results}

Candidate miRNAs targeting AKTs

A total of 29 miRNAs were predicted to respectively target AKT1, AKT2 and AKT3 by more than 3 out of 5 different programs (TargetScan, miRDB, DIANA-microT, miRanda and miRWalk) were listed in Additional file 1: Table S1. Then, RNAhybrid was used to calculate the hybridization free energy of the duplex miRNA:mRNA. The candidate miRNAs were thus confirmed according to the minimum free energy (MFE) values of their interactions with AKTs. As shown in Additional file 2: Table S2, 6 optimized

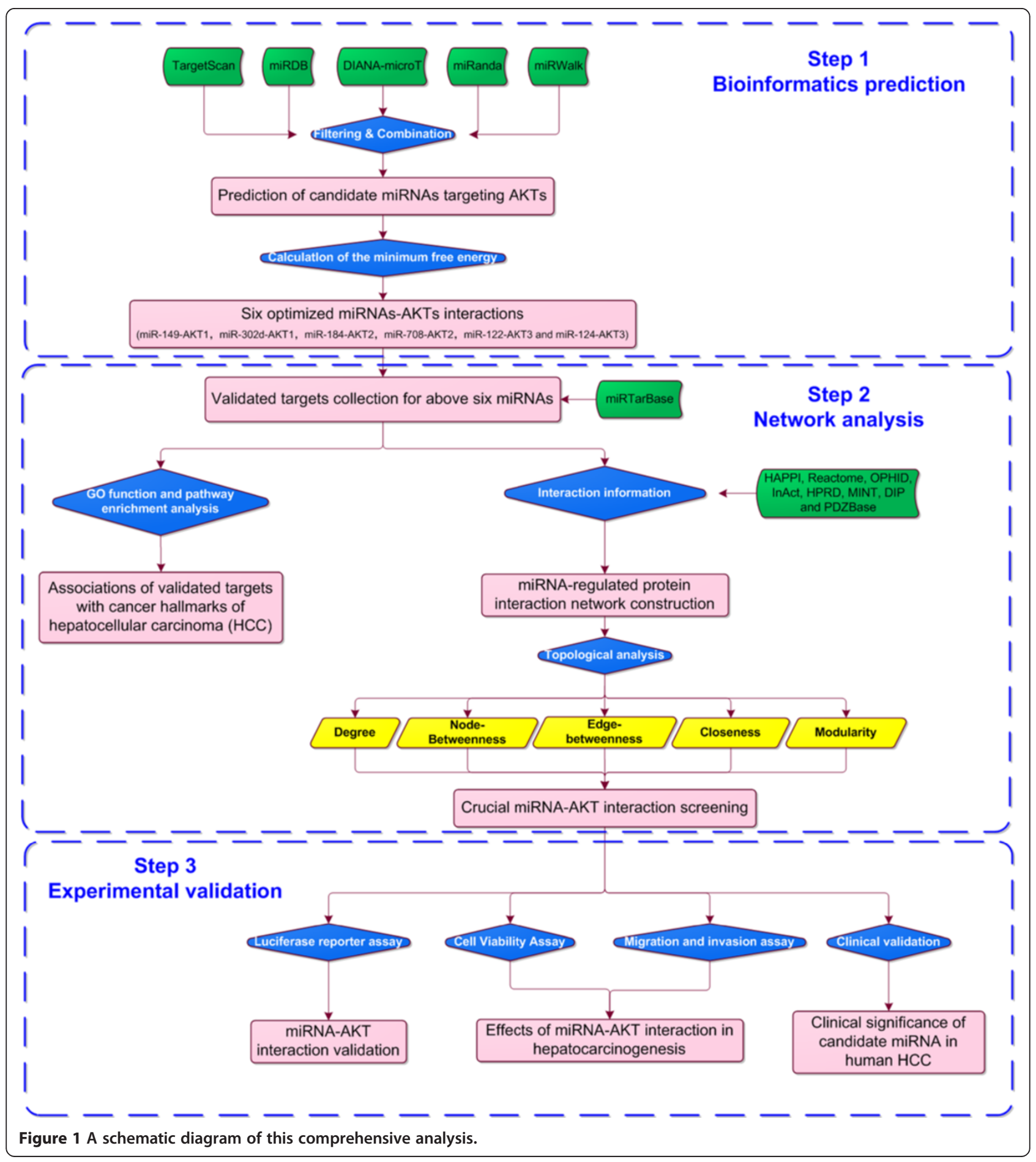


miRNA-AKT interactions (miR-149-AKT1, miR-302dAKT1, miR-184-AKT2, miR-708-AKT2, miR-122-AKT3 and miR-124-AKT3) had lower MFEs than the median values $[-33.35(\mathrm{kcal} / \mathrm{mol})]$ of all MFEs, thus, miR-149 and miR-302d were identified as candidate miRNAs targeting AKT1, miR-184 and miR-708 were identified as candidate miRNAs targeting AKT2, and miR-122 and miR-124 were identified as candidate miRNAs targeting AKT3.

\section{Enrichment analysis of validated targets for candidate miRNAs}

To elucidate the functions of 6 candidate miRNAs, 103 validated targets for them were collected from miRTarBase. Please see detail information on these validated targets in Additional file 3: Table S3. Then, the enrichment analysis based on GO annotation system was performed. This system uses a controlled and hierarchical vocabulary to assign function to genes or gene products in any organism. As shown in Additional file 4: Figure S1A, the validated targets for 6 candidate miRNAs had enriched GO terms related to apoptosis, cell death, and cell cycle. In terms of pathway information which is important for understanding gene and protein function, the validated targets were significantly enriched in many known oncogenic pathways for $\mathrm{HCC}$, such as focal adhesion, cell cycle, p53 signaling pathway, mTOR signaling pathway, apoptosis, VEGF signaling pathway, etc. (Additional file 4: Figure S1B).

\section{Network analysis}

The interaction information of proteins encoded by validated targets of 6 candidate miRNAs were used to construct candidate miRNAs-regulated protein interaction network, which consists of 2868 nodes and 4779 edges. Please see detail information on this network in Additional file 5: Table S4.

Hub proteins in the protein interaction networks have extremely high levels of degree and tend to encode essential genes. According to the previous studies of $\mathrm{Li}$ et al. [16] and our research group [15,17], we identified a node as a hub protein if its degree is more than 2 fold of the median degree of all nodes in a network. As a result, 113 nodes were identified as hub proteins. Then, we constructed the interaction network of these hub proteins, which consists of 99 nodes and 355 edges as shown in Figure 2A. Please see detail information on this network in Additional file 6: Table S5.

Three topological features, 'Degree', 'Node betweenness' and 'Closeness' (defined in 'Materials and methods' section) were chosen to identify topological important nodes. After calculating the value of the 3 features for each hub node, the median values of 'Degree', 'Node betweenness' and 'Closeness' were 7, 1.07 and 38.13, respectively. Therefore, we determined that hub nodes with
'Degree' > 7, 'Node betweenness' > 1.07, and 'Closeness' > 38.13 were topological important nodes. As a result, 28 topological important nodes were selected (Additional file 7: Table S6). Among them, 16 (AKT1, AKT2, AKT3, AR, CDK2, CDK4, CDK6, E2F1, IGF1R, MYBL2, NR3C1, PTPN1, RAC1, RELA, RHOA and SOCS1) were validated targets for candidate miRNAs targeting AKTs.

Modularity has been reported to be another important aspect of a protein interaction network. Nodes that are highly interconnected within the network are usually involved in the same biological modules or pathways. Using Markov clustering algorithm, we divided the interaction network of hub proteins into 5 functional modules containing 72, 10, 9, 4 and 4 nodes, respectively (Figure 2B). According to the enrichment analysis based on Gene Ontology (GO) annotation system and KEGG pathway, the biggest functional module was significantly associated with PI3K/AKT/mTOR signaling pathway, MAPK signaling pathway, cell cycle, focal adhesion and Wnt signaling pathway. The other modules were respectively involved in G1/S transition of mitotic cell cycle, regulation of gene expression, apoptosis and Notching signaling pathway.

Since an interaction with a high 'edge-betweenness' has been defined as a bottleneck which has many 'shortest paths' going through it and controls the rate of information flow, we further calculated the 'edge-betweenness' of each interaction in the interaction network of hub proteins. As shown in Additional file 8: Table S7, the interaction between AKT1-mTOR had the highest edge-betweenness value (164.38), suggesting its importance in connecting different modules in the network.

Taken together, the above network analysis showed that AKT1 and its interaction with mTOR respectively had the highest node-betweenness and edge-betweenness, implying their bottleneck roles in the network. According to the combination of the miRNA target prediction and MFE calculation, miR-149 and miR-302d were identified as candidate miRNAs targeting AKT1. Pan et al. [18] reported the regulatory effect of miR-149 on AKT1 in glioma cells. Lin et al. [19] also identified AKT1 as a direct target of miR-149* in various cancer cells, including neuroblastoma and Hela cells. However, the interaction between miR-149 and AKT1 has not been validated in HCC cells. In addition, the regulatory effect of miR-302d on AKT1 has not been reported previously. On this context, we would like to perform extensive experiments to confirm the miR149-AKT1-mTOR axis and its clinical relevance in human HCC.

\section{Experimental validation}

\section{MiRNA-149 directly targets AKT1 in HCC cells}

To verify the regulatory effect of miR-149 on AKT1 in HCC cells, we transfected HepG2 cells with miR-149 mimics, miR-149 mimic control (negative control, NC), 


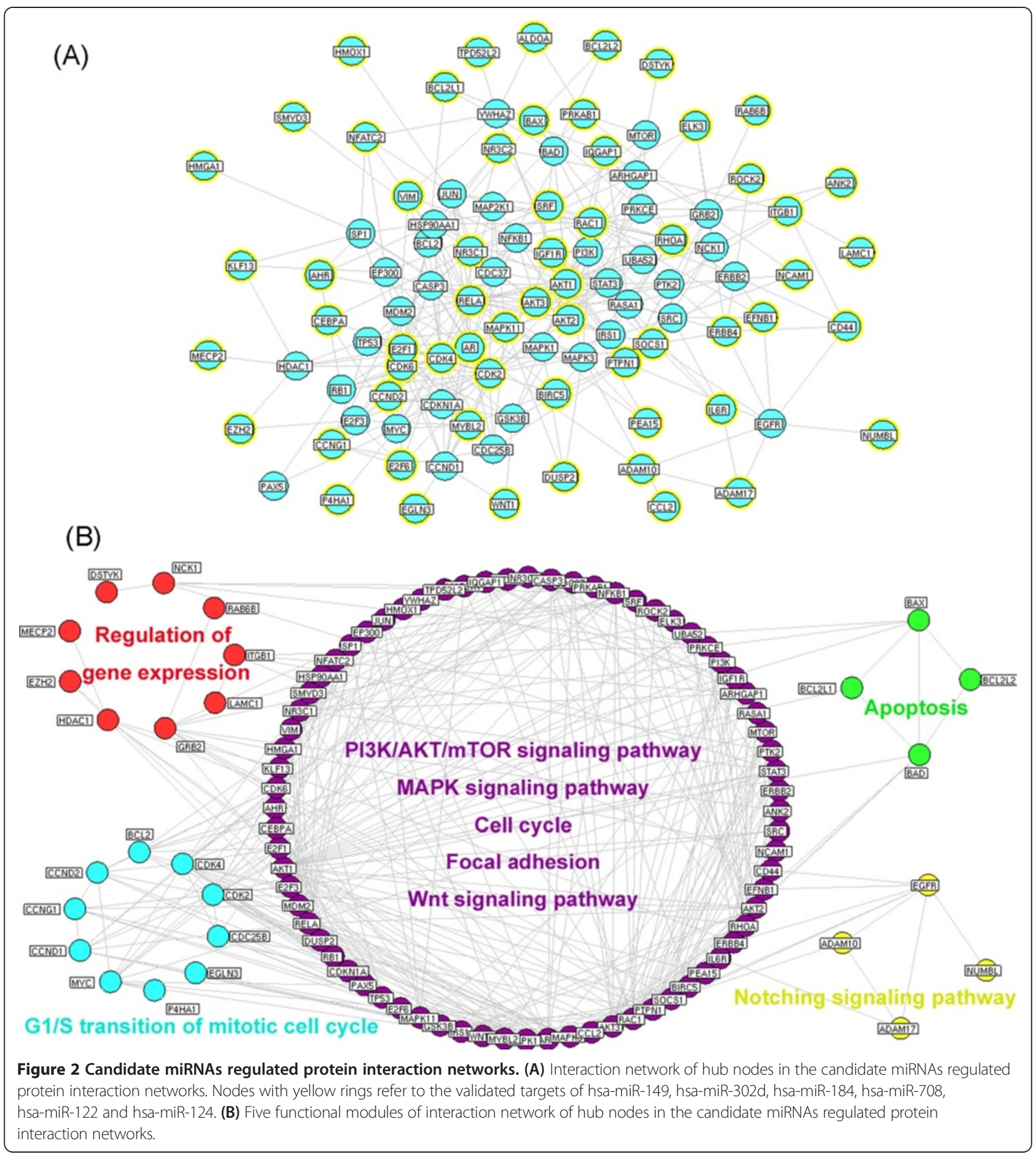

and blank control culture medium (mock). After $24 \mathrm{~h}$ post-transfection, the expression level of AKT1 protein in HepG2 cells overexpressed miR-149 was significantly lower than those in $\mathrm{NC}$ and mock groups (both $\mathrm{P}<0.001$, Figure $3 \mathrm{~A} \sim \mathrm{C}$ ).

To verify whether AKT1 was a direct target of miR-149, the luciferase reporter containing the complimentary seed sequence of miR-149 at the 3'-UTR region of AKT1
mRNA was constructed (Figure 3D). Luciferase activity was detected at $48 \mathrm{~h}$ after the co-transfection of FLuci vector (3'-UTR-AKT1wt FLuci vector or 3'-UTR-AKT1mut FLuci vector), miR-149 mimic or NC mimic, and RLuci vector in HepG2 cells. As shown in Figure 3E, the luciferase activity was significantly decreased in HepG2 cells co-transfected with 3'-UTR-AKT1wt FLuci vector and miR-149 mimic compared with those co-transfected with 


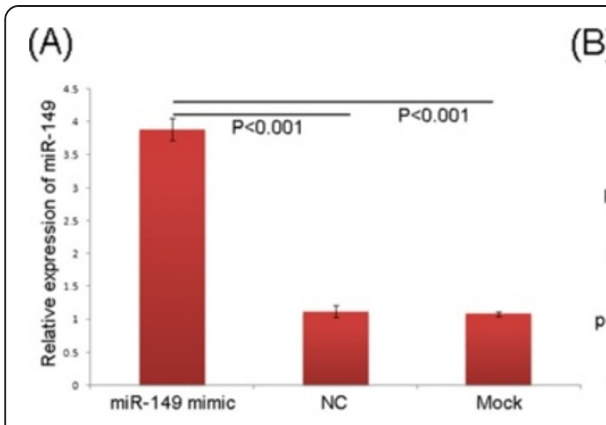

(D)

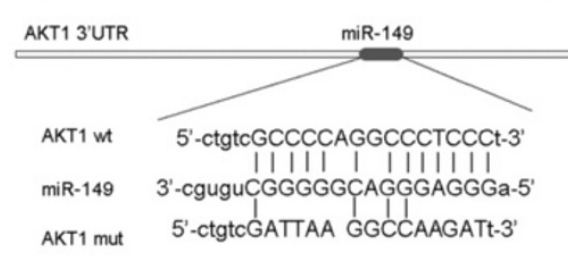

(B)

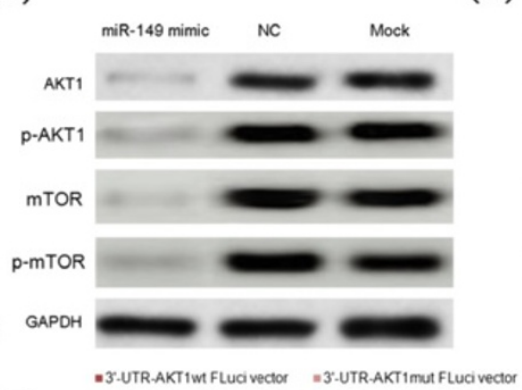

(E)

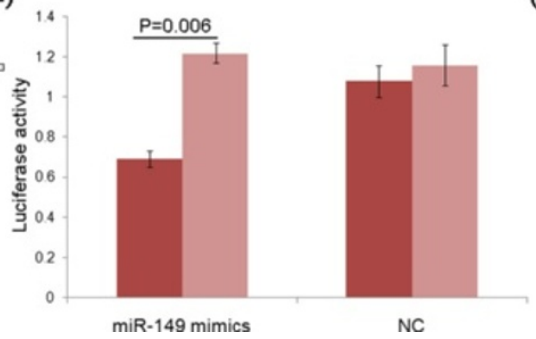

(C)

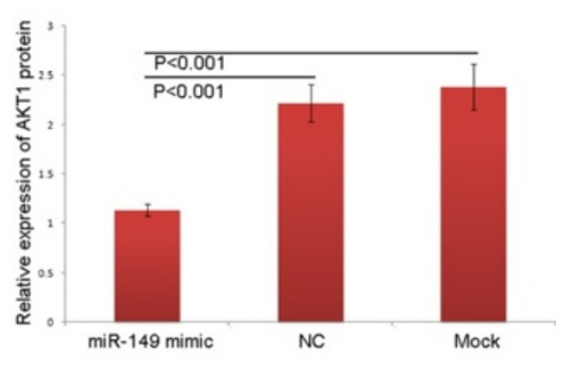

(F)

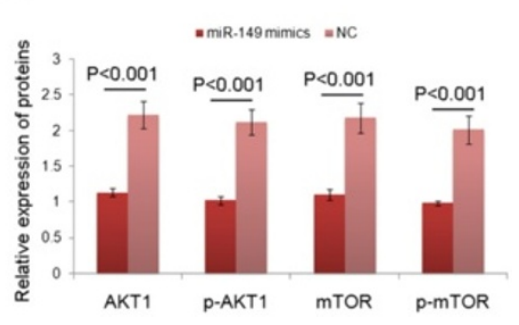

Figure 3 MiRNA-149 directly targets AKT1 in HCC cells. (A) QRT-PCR analysis showing relative expression of miR-149 in HepG2 cells transfected with miR-149 mimics, miR-149 mimic control (negative control, NC), and blank control culture medium (mock). (B, C and F) Relative expression of AKT1, p-AKT1, mTOR and p-mTOR proteins in HepG2 cells transfected with miR-149 mimics, miR-149 mimic control (negative control, NC), and blank control culture medium (mock) detected by Western blot analysis. GAPDH was used as an internal loading control. (D) RNA sequence alignment showing the 3'-UTR of AKT1 mRNA contains a complementary site for the seed region of miR-149. AKT1mut is amutant with substitutions in the complementary region as a negative control. (E) Luciferase report assay was performed to verify whether AKT1 was a direct target of miR-149. The luciferase activity was detected after transfection of FLuci vector (3'-UTR-AKT1wt FLuci vector or 3'-UTR-AKT1mut FLuci vector) into the miR-149 mimic or miR-149 mimic control (negative control, NC) transfected HepG2 cells.

3'-UTR-AKT1mut FLuci vector and miR-149 mimic $(P=0.006)$, suggesting that the fragment at the 3 '-UTR of the AKT1 mRNA was the complementary site for the miRNA-149 seed region, and thus, that AKT1 was a direct target of miR-149.

\section{MiR-149 regulates the AKT1/mTOR pathway in HCC cells} To verify the regulatory effect of miR-149 in AKT/mTOR pathway, we examined the expression of key components in this pathway, including AKT1, pAKT1, mTOR and pmTOR, in HepG2 cells with or without miR-149 overexpression. As shown in Figure $3 \mathrm{~B}$ and F, the total and phosphorylated protein expression levels of AKT1 and mTOR were all significantly decreased in HepG2 cells overexpressed miR-149 (all $\mathrm{P}<0.001$ ), indicating that miR-149 might be an important regulator of this signaling pathway.

\section{Reverse correlation between miR-149 and AKT1 mRNA expression in human HCC tissues and cells}

QRT-PCR was performed to evaluate the relationship between miR-149 and AKT1 mRNA expression in HCC tissues and cells in vitro. As shown in Figure 4A and B, the expression level of miR-149 in HCC tissues was significantly lower than that in adjacent nonneoplastic liver tissues $(\mathrm{P}<0.001$, Figure $4 \mathrm{~A})$, while AKT1 mRNA expression was dramatically increased in HCC tissues compared to adjacent nonneoplastic liver tissues $(\mathrm{P}<0.001$, Figure $4 \mathrm{~B}$ ), which was similar to our previous study on the expression levels of AKT1 protein by immunohistochemistry (IHC) analysis based on the same cohort of HCC patients (IHC scores of AKT1 protein in HCC tissues vs. adjacent nonneoplastic liver tissues, mean \pm S.D.: $6.32 \pm$ 1.74 vs. $2.48 \pm 0.29, \mathrm{P}<0.001$ ) [15]. More interestingly, the Spearman Correlation analysis clearly showed negative correlations between miR-149 and AKT1 mRNA (protein, IHC scores) expression in HCC tissues (for miR-149 and AKT1 mRNA: rs $=-0.639, \mathrm{P}<0.001$, Figure $4 \mathrm{C}$; for miR149 and AKT1 protein: $r s=-0.716, \mathrm{P}<0.001$, Figure 4D). These findings were consistent with those based on HCC cells in vitro system (Figure $4 \mathrm{E} \sim \mathrm{F}$ ).

MiR-149 inhibits cell proliferation, invasion and migration of HCC cells in vitro by targeting AKT1/mTOR pathway

The down-regulation of miR-149 in HCC cells and tissues prompted us to verify whether it acted as a tumor suppressor in this malignancy. As shown in Figure 5A, the enforced expression of miR-149 significantly inhibited cell proliferation of HepG2 cells $(\mathrm{P}=0.01)$. In addition, transwell assays revealed that overexpression of miR-149 could dramatically reduce the invasion activities of HepG2 cells compared with those of control cells ( $\mathrm{P}=0.008$, Figure 5B and $\mathrm{C}$ ). Moreover, wound-healing assays also demonstrated 


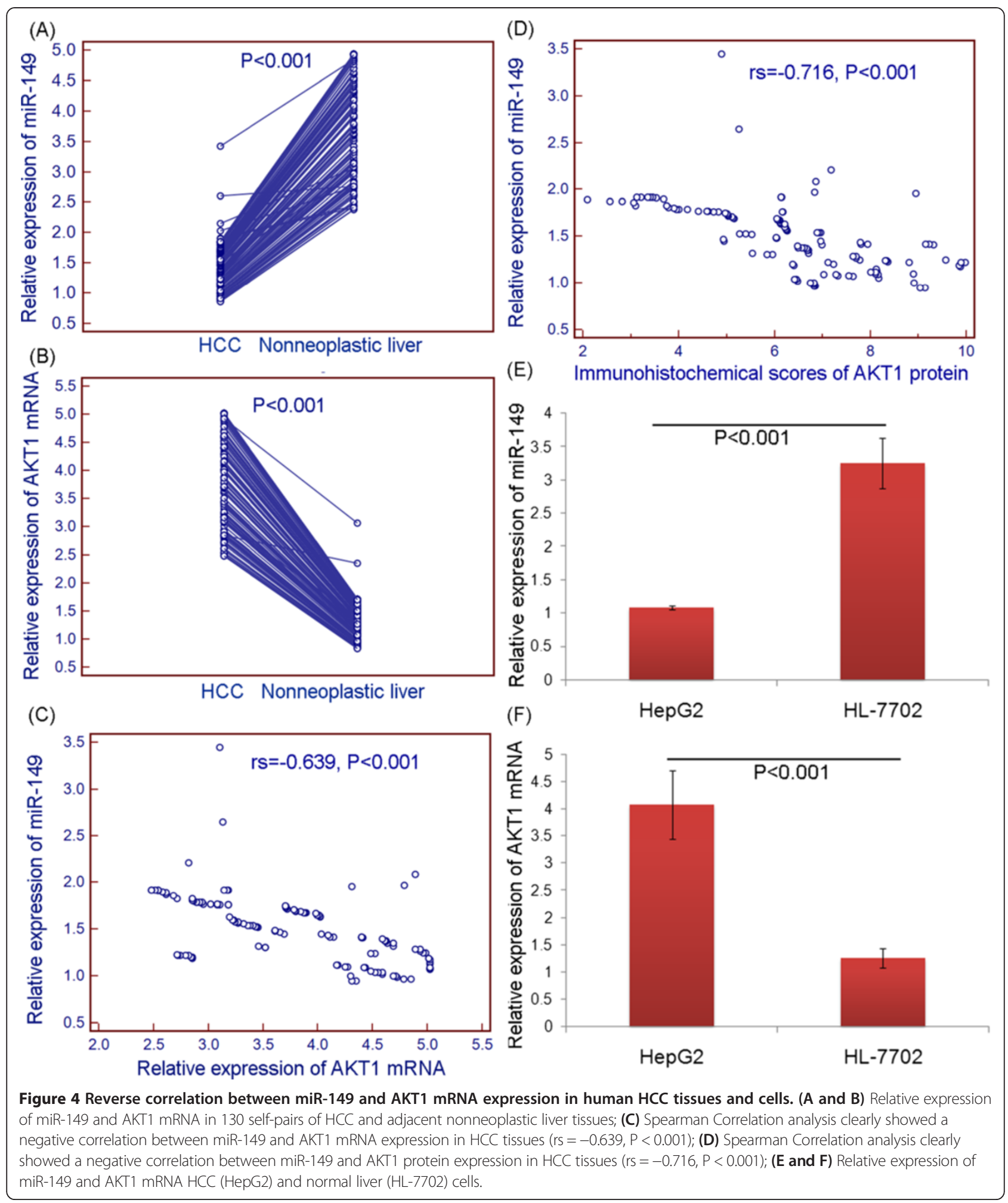

that overexpression of miR-149 could markedly reduce the migration activities of HepG2 cells $(P=0.01$, Figure 5D and E).

To further verify whether the role of miR-149 in tumorigenesis of $\mathrm{HCC}$ was mediated via targeting AKT1-mTOR signaling, AKT1 expression in HepG2 cells was knockdown via siRNA, which resulted in the downregulation of $\mathrm{AKT} 1, \mathrm{p}-\mathrm{AKT} 1, \mathrm{mTOR}$ and mTOR proteins (Figure 6A and B). Furthermore, knockdown of AKT1 expression abolished effects of miR-149 overexpression in HepG2 


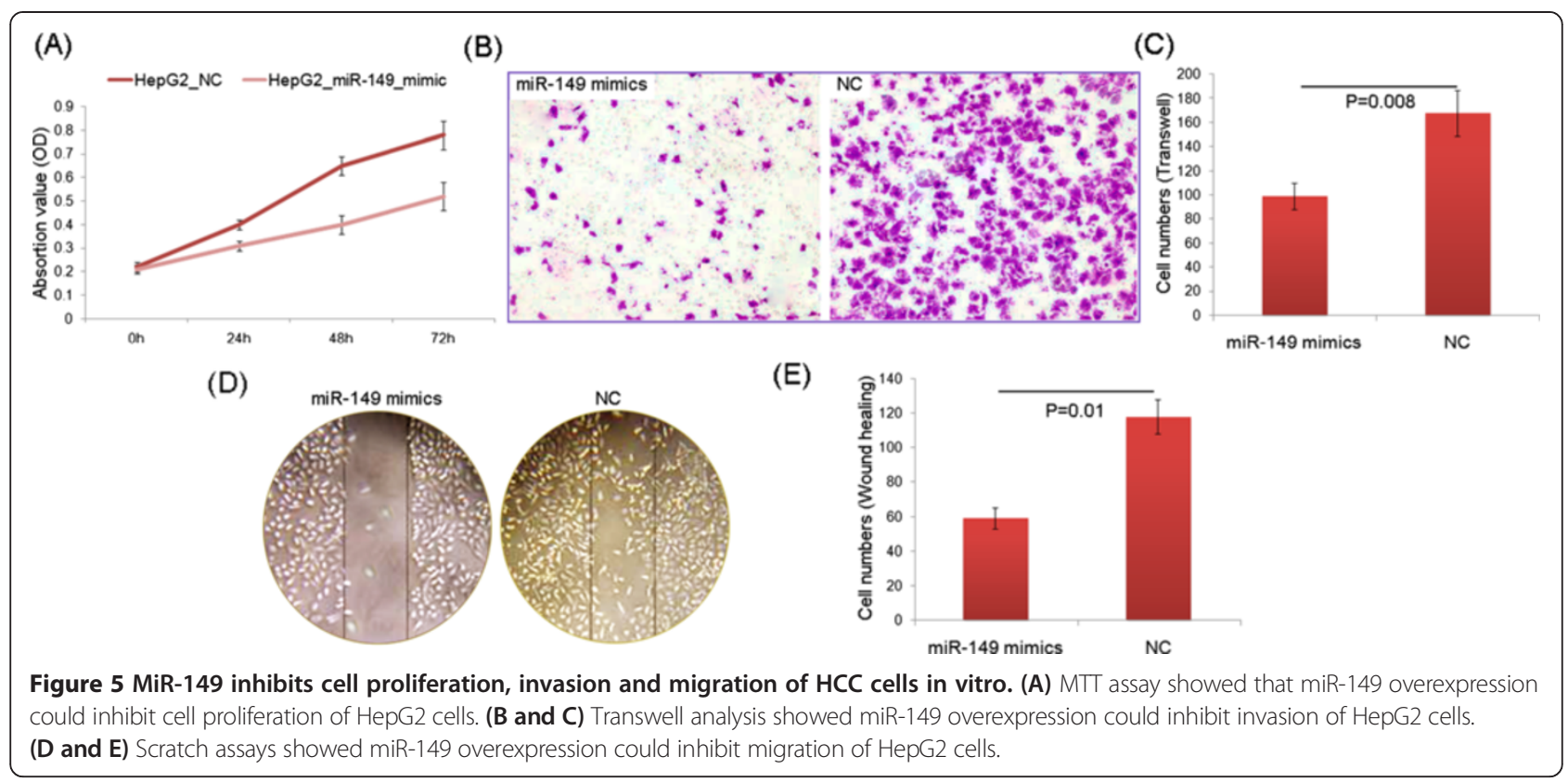

cells, including inhibiting cell proliferation (Figure 6C), invasion (Figure 6D) and migration (Figure 6E), indicating that the tumor suppressive roles of miR-149 in HCC were partially mediated by targeting AKT1-mTOR pathway.

\section{Down-regulation of miR-149 associates the advanced} tumor progression and poor prognosis of human HCC

To evaluate whether miR-149 expression was associated with clinicopathological features of patients with HCC, we analyzed the association of miR-149 expression with
T stage, tumor grade, presence of cirrhosis, underlying liver disease including alcohol abuse, viral hepatitis B and C, sex, and age (Table 1). In order to classify all 130 HCC patients into high miR-149 expression and low miR-149 expression groups, a cutoff value for miR-149 expression levels was chosen on the basis of a measure of heterogeneity with the log-rank test statistic with respect to overall survival. As a result, there were 70 (70/ $130,53.85)$ patients belonging to low miR-149 expression group and $60(60 / 130,46.15 \%)$ patients belonging
(A)

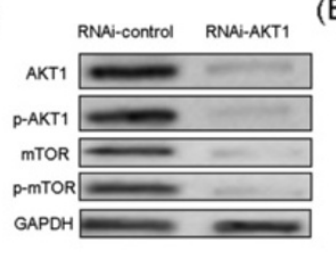

(D)

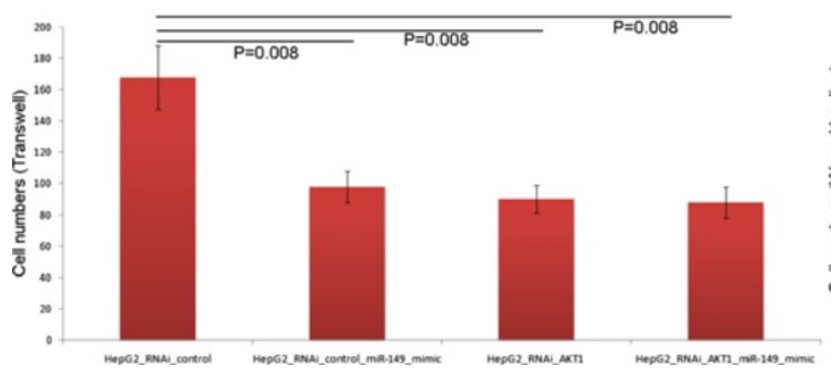

(B)

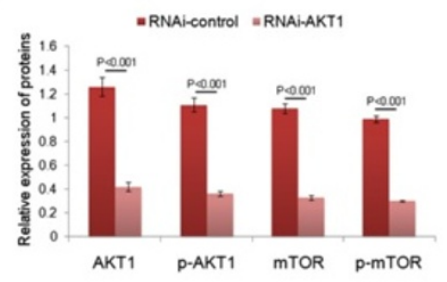

(C)

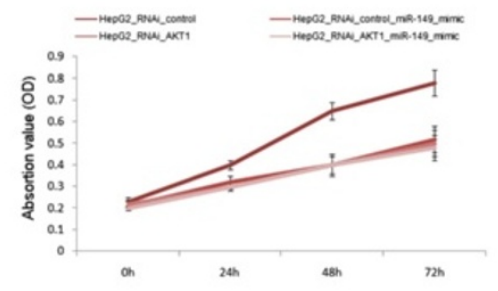

(E)

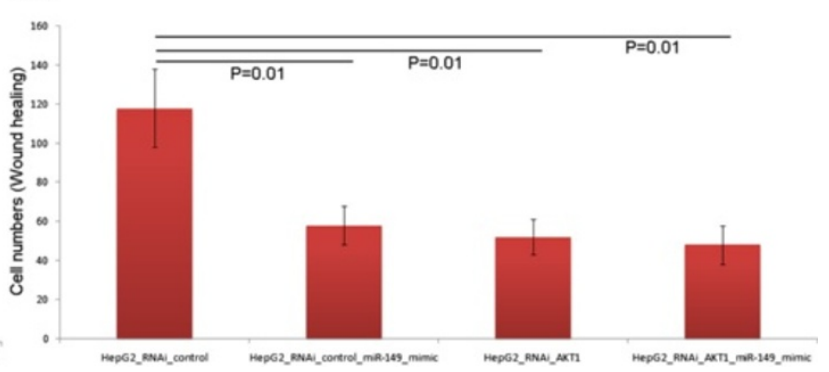

Figure 6 MiR-149 inhibits cell proliferation, invasion and migration of HCC cells in vitro by targeting AKT1/mTOR pathway. (A and B) Western blot analysis of AKT1, p-AKT1, mTOR and mTOR protein expression levels in HepG2 cells transfected with RNAi-AKT1 or RNAi-control vectors. (C-E) Overexpression of miR-149 fails to inhibit proliferation, invasion, and migration in AKT1 knockdown HepG2 cells. 
Table 1 Association of miR-149 expression with clinicopathologic features of $\mathbf{1 3 0}$ hepatocellular carcinoma patients

\begin{tabular}{|c|c|c|c|}
\hline Clinicopathologic features & Case & miR-149-low (n, \%) & $\mathbf{P}$ \\
\hline \multicolumn{4}{|l|}{ Age (years) } \\
\hline$\leq 50$ & 72 & $38(52.78)$ & \multirow[t]{2}{*}{ NS } \\
\hline$>50$ & 58 & $32(55.17)$ & \\
\hline \multicolumn{4}{|l|}{ Gender } \\
\hline Male & 96 & $50(52.08)$ & \multirow[t]{2}{*}{ NS } \\
\hline Female & 34 & $20(58.82)$ & \\
\hline \multicolumn{4}{|l|}{ Serum AFP } \\
\hline Positive & 72 & $42(58.33)$ & \multirow[t]{2}{*}{ NS } \\
\hline Negative & 58 & $28(48.28)$ & \\
\hline \multicolumn{4}{|l|}{ Tumor stage } \\
\hline T1 & 23 & $6(26.09)$ & \multirow[t]{4}{*}{0.01} \\
\hline $\mathrm{T} 2$ & 40 & $15(37.50)$ & \\
\hline T3 & 52 & $34(65.38)$ & \\
\hline T4 & 15 & $15(100.00)$ & \\
\hline \multicolumn{4}{|l|}{ Tumor grade } \\
\hline G1 & 31 & $16(51.61)$ & \multirow[t]{3}{*}{ NS } \\
\hline G2 & 76 & $39(51.32)$ & \\
\hline G3 & 23 & $15(65.22)$ & \\
\hline \multicolumn{4}{|l|}{ Growth pattern } \\
\hline Trabecular & 101 & $53(52.48)$ & \multirow[t]{2}{*}{ NS } \\
\hline Nontrabecular & 29 & $17(58.62)$ & \\
\hline \multicolumn{4}{|l|}{ Cirrhosis } \\
\hline Yes & 86 & $45(52.33)$ & \multirow[t]{2}{*}{ NS } \\
\hline No & 44 & $25(56.82)$ & \\
\hline \multicolumn{4}{|l|}{ Underlying liver disease } \\
\hline Alcoholic & 25 & $13(52.00)$ & \multirow[t]{4}{*}{ NS } \\
\hline Hepatitis B & 49 & $28(57.14)$ & \\
\hline Hepatitis C & 35 & $17(48.57)$ & \\
\hline Unknown & 21 & $12(57.14)$ & \\
\hline
\end{tabular}

Note: 'NS' refers to the differences among groups have no statistical significance.

to low miR-149 expression group. Statistical analysis showed that the down-regulation of miR-149 was more frequently found in HCC tissues with high tumor stage (T3 4) than those with low tumor stage (T1 2, $\mathrm{P}=0.01$, Table 1).

To determine the prognostic value of miR-149 in patients with HCC, the Kaplan-Meier method was employed to analyze the correlation between miR-149 expression with 5-year disease-free survival and 5-year overall survival of HCC patients. As shown in Figure 7A, we observed a trend that 5-year disease-free survival of HCC patients with low miR-149 expression was significantly poorer than those with high miR-149 expression $(\mathrm{P}<0.001$, log-rank test; Figure 7A). Similarly, the Kaplan-Meier plot of 5-year overall survival curves stratified by miR-149 expression also showed a significant relationship between miR-149 expression and 5-year overall survival $(\mathrm{P}<0.001$, log-rank test, Figure 7B). Furthermore, the multivariate analysis found that miR-149 expression was an independent poor prognostic factor for both 5-year disease-free survival $(\mathrm{P}=0.003$, Table 2$)$ and 5 -year overall survival $(\mathrm{P}=0.006$, Table 2) in HCC.

\section{Discussion}

Elucidating the molecular mechanisms of tumor progression and tumor prognosis in human HCC remains largely unexplored. In the current study, we identified 6 candidate miRNAs targeting AKTs using an improved prediction protocol with two steps: (1), 5 different programs for miRNA target prediction were used due to their various algorithms and we identified the overlap which was the consensus among 3 out of 5 different programs; (2), we selected miRNA-target interactions (MTIs) with low hybridization energies. These two steps were performed to minimize false positive and false negative results. According to the enrichment analysis on GO items and KEGG pathways, we found that the most common functions of the experimentally validated targets of candidate miRNAs targeting AKTs included focal adhesion, cell cycle, p53 signaling pathway, mTOR signaling pathway, apoptosis, VEGF signaling pathway, which cover all the hallmarks of HCC, providing a convincing evidence that these candidate miRNAs may have a definitive impact on hepatocarcinogenesis. In order to improve our understanding of this candidate miRNAs on HCC malignant progression, we constructed the protein interaction network of their validate targets. Following the network topological analysis, we found that AKT1 and its interaction with mTOR respectively had the highest node-betweenness and edgebetweenness. Many previous studies have recognized highly connected nodes (hubs) as the most important points in the network. Very recently, growing reports have put forward a complementary notion that is bottlenecks as nodes/edges with a high betweenness centrality (i.e., network nodes/edges which have many 'shortest paths' going through them). In fact, bottlenecks represent key connector proteins/interactions with surprising functional and dynamic properties [20]. On this context, AKT1mTOR may be the most important interaction in our miRNAs-regulated protein interaction network. Thus, we further performed a systematic experiments to validate the regulatory effect of a candidate miRNA targeting AKT1-miR-149 on AKT1-mTOR axis, and its involvement in hepatocarcinogenesis and clinical progression of HCC.

MiR-149, located on chromosome 2 [21], has been reported to play controversial roles in the progression of various types of human cancers. It is downregulated in 


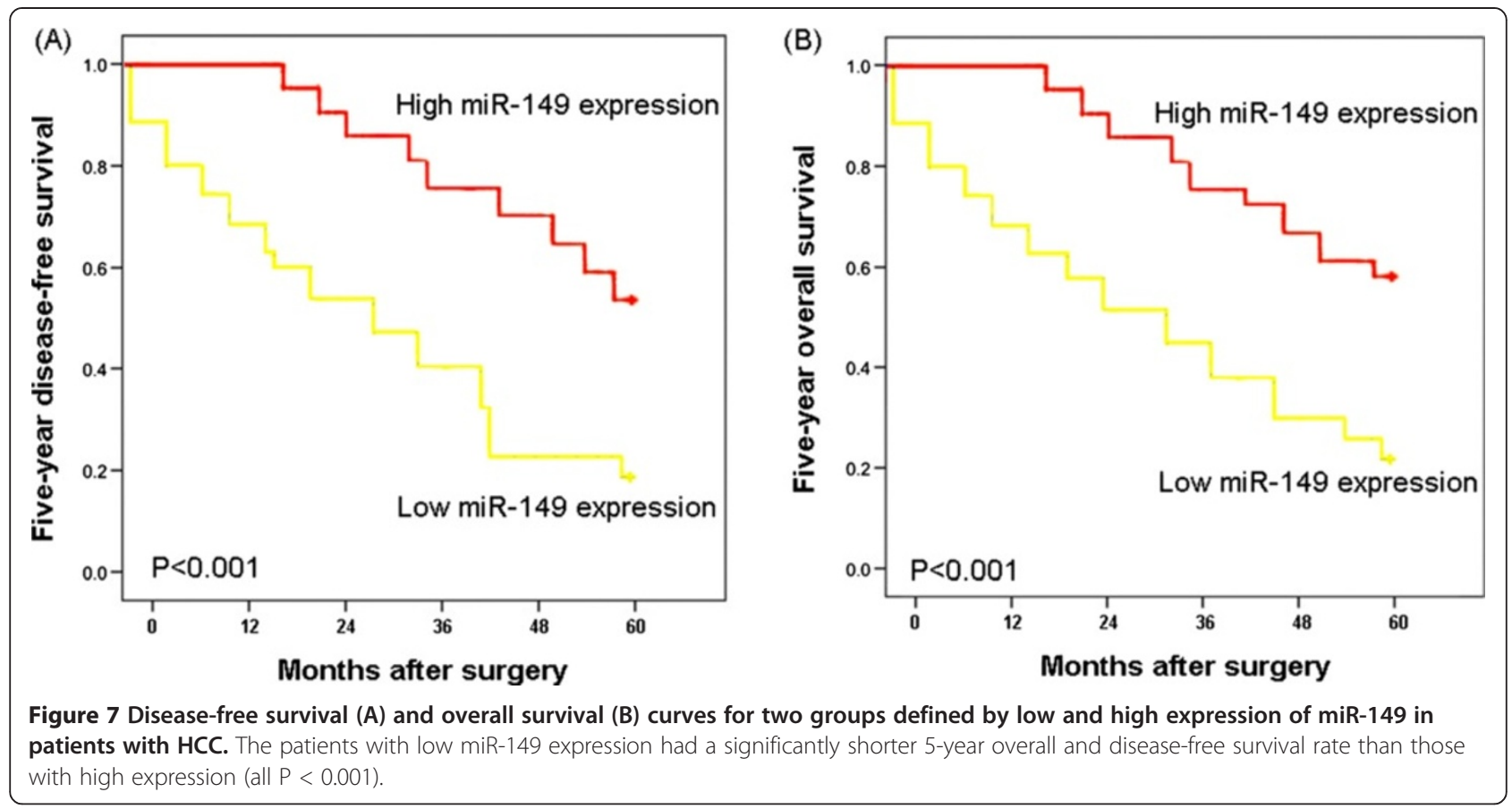

several cancer cells and functions as a tumor suppressor by targeting oncogenes. For example, Chan et al. [22] reported that miR-149 could suppress breast cancer cell migration/invasion and metastasis by targeting GIT1; Cheng et al. [23] observed the decreased expression of miR-149 in clear cell renal cell carcinoma; Ke et al. [24] found that the expression of miR-149 was downregulated in lung cancer and miR-149 could inhibit EMT by targeting FOXM1; Pan et al. [18] indicated that miR-149 may play a tumor suppressive role in the proliferation and invasion of glioma cells via blockade of the AKT1 signaling. In contrast, miR-149 is also upregulated in several cancer cells and functions as a oncomiRs by targeting certain tumor suppressive genes. For example, miR-149 was highly expressed in nasopharyngeal carcinoma and promoted malignant progression by suppressing the expression of its target gene, Smad2 [25]; Its oncogenic role was also demonstrated in melanoma in which miR-149 upregulation caused the downregulation of GSK3- and upregulation of $\mathrm{Mcl}-1$, leading to apoptotic resistance [26]. According to miRTarBase (Release 4.5: Nov. 1, 2013; http://mirtarbase.mbc.nctu.edu.tw/), E2F1 and MYBL2, together with AKT1, are validated targets for miR-149. Chen and colleagues reported that the upregulation of E2F1 protein might associate with worse outcomes in patients with HCC [27]. Frau and colleagues also indicated that MYBL2 upregulation could induce fast growth and progression of premalignant and malignant liver, through cell cycle deregulation and activation of genes and pathways related to tumor progression [28]. Nakajima and colleagues identified MYBL2 as a probable transcriptional target of E2F1 in HCC and as a useful biomarker for diagnosis and an attractive target for molecular therapies useful to treat HCC [29]. In the present study, our data

Table 2 Multivariate survival analysis of five-year overall and disease-free survival in 130 patients with hepatocellular carcinoma

\begin{tabular}{|c|c|c|c|c|c|c|}
\hline \multirow[t]{2}{*}{ Features } & \multicolumn{3}{|c|}{ Five-year overall survival } & \multicolumn{3}{|c|}{ Five-year disease-free survival } \\
\hline & HR & $95 \% \mathrm{Cl}$ & $P$ & HR & $95 \% \mathrm{Cl}$ & $\mathbf{P}$ \\
\hline Age & 1.132 & $0.316-3.516$ & 0.192 & 1.536 & $0.322-3.736$ & 0.125 \\
\hline Gender & 1.191 & $0.345-3.857$ & 0.136 & 1.559 & $0.357-3.831$ & 0.131 \\
\hline Serum AFP & 1.931 & $0.685-4.056$ & 0.063 & 1.953 & $0.615-4.273$ & 0.062 \\
\hline Tumor stage & 2.879 & $1.366-5.196$ & 0.009 & 2.686 & $1.386-6.009$ & 0.01 \\
\hline Tumor grade & 1.563 & $0.609-4.088$ & 0.081 & 1.551 & $0.607-4.466$ & 0.086 \\
\hline Presence of cirrhosis & 1.919 & $0.738-4.102$ & 0.063 & 1.921 & $0.793-4.219$ & 0.062 \\
\hline miR-149 expression & 3.938 & $1.472-8.038$ & 0.003 & 3.608 & $1.431-7.686$ & 0.006 \\
\hline
\end{tabular}


showed the downregulated expression of miR-149 in HCC cell line and clinical specimens compared with the normal liver cell and matched adjacent nonneoplastic liver tissues, respectively. In addition, our functional study showed that AKT1 was a downstream target and effector of miR-149. Enforced expression of miR-149 and knockdown of AKT1 both inhibited the cell proliferation and tumorigenicity of HCC cell line in vitro. Strikingly, miR-149 overexpression did not inhibit the cell proliferation and tumorigenicity of HCC cells in vitro when AKT1 was knocked down. Then, our investigation of the downstream effectors of miR-149AKT1 signaling axis showed that enforced expression of miR-149 and knockdown of AKT1 both could impair the activation of AKT1 and mTOR. Our data here provide the first and direct evidence that miR-149 has an tumor suppressive role in HCC cells by regulating AKT1/mTOR signaling, which was similar with the previous findings of Pan et al. [18] on glioma cells. More importantly, our data showed that the expression level of miR-149 was associated with tumor stage of HCC patients. Advance tumor stage samples have lower miR-149 expression compared to early tumor stage samples. These findings were similar to the data of Wang et al. on gastric cancer [30]. Furthermore, we also identified miR-149 as an independent prognostic factor for both 5-year disease-free survival and 5-year overall survival of $\mathrm{HCC}$ patients.

\section{Conclusion}

This comprehensive analysis identified a list of miRNAs targeting AKTs and revealed their critical roles in HCC malignant progression. Especially, miR-149 may function as a tumor suppressive miRNA and play an important role in inhibiting the $\mathrm{HCC}$ tumorigenesis by modulating the AKT/mTOR pathway. Our clinical evidence also highlight the prognostic potential of miR-149 in HCC. The newly identified miR-149/AKT/mTOR axis might be a promising therapeutic target in the prevention and treatment of HCC.

\section{Materials and methods}

\section{Prediction of miRNAs targeting AKTs}

Candidate miRNAs which target AKT1, AKT2 and AKT3 were predicted by five different online programs, including TargetScan (Release 6.2, http://www.targetscan.org/) [31], miRDB (Last modified: April 03, 2012. http://mirdb.org/ miRDB/) [32], DIANA-microT (DIANA-microT-CDS v5.0, http://diana.imis.athena-innovation.gr/ DianaTools/index. php? $=$ microT_CDS/index) [33], miRanda (Last Update: 2010-11-01, http://www.microrna.org/microrna/home.do) [34] and miRWalk (Last Update: 15th March 2011. http:// www.umm.uni-heidelberg.de/apps/zmf/mirwalk/) [35], which represent different algorithms based on diverse features, thus, their combination could ensure high specificity of the prediction. miRNAs which were commonly predicted by more than 3 out of 5 programs were retained.

Then, RNAhybrid [36] was used to calculate the MFE of the duplex miRNA:mRNA. We chose miRNA-AKTs interactions, MFE values of which were lower than the median of all MFE values, as candidate miRNA-AKTs interaction.

After that, validated targets for above candidate miRNAs which target AKTs were collected from miRTarBase (Release 4.5: Nov. 1, 2013; http://mirtarbase.mbc.nctu. edu.tw/), which has accumulated more than fifty thousand MTIs [37]. Generally, the collected MTIs are validated experimentally by reporter assay, western blot, microarray and next-generation sequencing experiments. Here, we only collected the MTIs which are validated experimentally by reporter assay, western blot and qPCR. In order to facilitate data analysis, the different ID types for validated target genes were converted to ID from UniProtKB-Swiss-Prot (release-2014_02).

\section{GO and pathway enrichment analysis for validated genes of candidate miRNAs which target AKTs}

We used Database for Annotation, Visualization and Integrated Discovery [38] (DAVID, http://david.abcc.ncifcrf. gov/home.jsp,version 6.7) for GO enrichment analysis. We also performed pathway enrichment analysis using pathway data obtained from the FTP service of KEGG [39] (Kyoto Encyclopedia of Genes and Genomes, http:// www.genome.jp/kegg/, Last updated: Oct 16, 2012).

\section{Network analysis}

\section{Protein-protein interaction (PPI) data}

PPI data were imported from eight existing PPI databases including Human Annotated and Predicted Protein Interaction Database (HAPPI) [40], Reactome [41], Online Predicted Human Interaction Database (OPHID) [42], InAct [43], Human Protein Reference Database (HPRD) [44], Molecular interaction Database (MINT) [45], Database of Interacting Proteins (DIP) [46], and PDZBase [47]. The detailed information on these PPI databases is described in Additional file 9: Table S8.

\section{Network construction}

Interactions between proteins encoded by validated target genes of candidate miRNAs which target AKTs were used to construct miRNA-regulated protein interaction networks. The PPI data were obtained from eight existing PPI databases as mentioned above. Then, we applied Navigator software (Version 2.2.1) and Cytoscape (Version 2.8.1) to visualize the networks.

\section{Defining network topological feature set}

For each node i in miRNA-regulated protein interaction network, we defined four measures for assessing its 
topological property: (1) 'Degree' is defined as the number of links to node i; (2) 'Node betweenness' is defined as the number of shortest paths between pairs of nodes that run through node i. (3) 'Closeness' is defined as the inverse of the farness which is the sum of node $\mathrm{i}$ distances to all other nodes. The Closeness centrality can be regarded as a measure of how long it will take to spread information from node $i$ to all other nodes sequentially. Degree, betweenness and closeness centralities can measure a protein's topological importance in the network. The larger a protein's degree/node betweenness/closeness centrality is, the more important the protein is in the PPI network [48]. (4) 'Modularity': proteins that are highly interconnected within the network are usually involved in the same biological modules or pathways. Here, we used a Markov clustering algorithm to divide all nodes into different functional modules.

For each edge in miRNA-regulated protein interaction network, we calculated its 'Edge Betweenness' to assess the importance of a specific interaction in the network. 'Edge Betweenness' is defined as the frequency of an edge that places on the shortest paths between all pairs of vertices in network [49]. The edges with highest betweenness values are most likely to lie between functional modules.

\section{Experimental validation \\ Patients and Tissue Samples}

The study was approved by the Research Ethics Committee of 302 Hospital of PLA, Beijing, China. Written informed consent was obtained from all patients. All specimens were handled and made anonymous according to the ethical and legal standards.

A total of 130 self-pairs of HCC specimens and adjacent nonneoplastic liver tissues were snap-frozen in liquid nitrogen and stored at $-80^{\circ} \mathrm{C}$ following surgery for qRTPCR assay. All the tissues were obtained from 130 patients with primary $\mathrm{HCC}$ who underwent a curative liver resection at the 302nd Hospital of PLA, Beijing, China. These patients were diagnosed as HCC between 2001 and 2006. None of the patients recruited in this study had chemotherapy or radiotherapy before the surgery. HCC diagnosis was based on World Health Organization (WHO) criteria. Tumor differentiation was defined according to the Edmondson grading system. Liver function was assessed using the Child-Pugh scoring system. Tumor staging was determined according to the sixth edition of the tumornode-metastasis (TNM) classification of the International Union against Cancer. The clinicopathological features of 130 patients are summarized in Table 1 .

The median follow-up period was 8.6 years. Postoperative surveillance included routine clinical and laboratory examinations every third month, computed tomography scans of the abdomen, and radiographs of the chest every third month. After 5 years, the examination interval was extended to 12 months.

\section{Cell culture}

Human HCC cell line HepG2 was obtained from the American Type Culture Collection (Manassas, VA, USA) and was cultured in DMEM (Invitrogen, USA) supplemented with $10 \%$ fetal bovine serum (Gibico, USA), $2 \mathrm{mM}$ L-glutamine and antibiotics. Normal human liver cell line HL-7702 was obtained from the American Type Culture Collection (Manassas, VA, USA) and was maintained in RPMI 1640 medium (Invitrogen, USA) supplemented with $10 \%$ fetal bovine serum (Gibico, USA). Two cell lines were both maintained at $37^{\circ} \mathrm{C}$ in a humidified chamber supplemented with $5 \% \mathrm{CO}_{2}$.

\section{Construction of miR-149 expression vectors and cellular transfection}

Commercial miR-149 mimics and miR-149 mimic control expression vectors were purchased from Invitrogen Life Technologies, USA. The sequence information of two vectors were as following: for miR-149 mimic, 5'-UCU GGC UCC GUG UCU UCA CUC CC-3', for miR-149 mimic control, 5' -GGG AGU GAA GAC ACG GAG CCA GA-3'. HepG2 cells were transfected with miR-149 mimics and miR-149 mimic control expression vectors with Lipofectamine 2000 reagent (Invitrogen Life Technologies, USA) according to the manufacturer's protocol.

\section{QRT-PCR}

The qRT-PCR analysis for miRNA and mRNA was performed according to the similar protocol of our previous studies [13,50]. U6 small RNA and GAPDH were respectively used as internal controls for normalization and quantification miR-149 and AKT1 expression. The primer sequences were listed in Additional file 10: Table S9. Relative quantification of miRNA and mRNA expression was evaluated using the comparative cycle threshold (CT) method. All experiments were done in triplicate. Mean normalized gene expression \pm SE was calculated from independent experiments.

\section{RNA interference and cellular transfection}

To knockdown the expression of the human Akt1 gene, pGCSIL-GFP-Akt1 small hairpin RNA (NM_005163), an Akt1-RNA interference (RNAi) lentiviral vector (RNAiAKT1), was constructed (Shanghai GeneChem Co, Ltd., Shanghai, China). The sense primer was 5'-CCGGGA GGCCAAGTCCTTGCTTTCAttcaagagaTGAAAGCAAG GACTTGGC- CTCTTTTTG-3', and the anti-sense primer was $5^{\prime}$-AATTCAAAAAGAGGCCAAGT-CCTTG CTTTCAtctcttgaaTGAAAGCAAGGACTTGGCCTC- 3. A scrambled short-hairpin RNA was used as a negative control (RNAi-control). HepG2 cells were seeded into 
6-well plates and incubated overnight, and then transfected using LipofectamineTM 2000 transfection reagent (Invitrogen) according to the manufacturer's instructions. The expression levels of AKT1 in HepG2 cells transfected with RNAi-AKT1 and RNAi-control were detected by Western blot analysis as described in the next section.

\section{Western blot}

The Western blot protocol and semiquantitative analysis were carried out following the protocol of our previous studies $[13,50]$. The specific antibodies were as following: AKT1 antibody (\#sc-1618, goat polyclonal antibody, dilution 1:150, Santa Cruz Biotechnology, Inc. USA), p-AKT1 (Ser 473) antibody (\#sc-7985, rabbit polyclonal antibody, dilution 1:150, Santa Cruz Biotechnology, Inc. USA), mTOR antibody (\#sc-1549, goat polyclonal antibody, dilution 1:100, Santa Cruz Biotechnology, Inc. USA), p-mTOR (Ser 2448) antibody (\#sc-101738, rabbit polyclonal antibody, dilution 1:100, Santa Cruz Biotechnology, Inc. USA), and GAPDH antibody (CW0266, dilution 1:1,000, CoWin Biotech). All experiments were done in triplicate. Mean normalized gene expression $\pm \mathrm{SE}$ was calculated from independent experiments.

\section{Luciferase reporter assay}

The regulatory effect of miR-149 to AKT1 was evaluated by a luciferase reporter assay in HepG2 cells following the protocol of our previous studies [13,50]. Briefly, the human AKT1 3'-UTR luciferase reporter construct was generated by cloning AKT1 3'-UTR sequence containing the predicted miR-149 binding site into the 3 '-UTR region of the pGL3 luciferase reporter vector (Promega Corporation, Madison WI, USA). The miR-149 binding site-deleted AKT1 3'-UTR luciferase reporter construct was generated by PCR fragments of AKT1 3'-UTR luciferase reporter construct lacking the target site and ligated. HepG2 cells were cultivated in 24-well plates and co-transfected using Fugene (Roche) with $100 \mathrm{ng}$ of pGL3-AKT1-miR-149 constructs, $10 \mathrm{ng}$ miR-149 mimic or NC mimic, and 2 ng pRL-SV40 RLuci vector (Promega). The luciferase activity assay was performed $24 \mathrm{~h}$ after transfection using the dual-luciferase reporter assay system (Promega Corporation, Madison WI) according to the manufacturer's instructions.

\section{In vitro cell proliferation assay}

The in vitro cell proliferation of HepG2 cells transfected with miR-149 mimic, miR-149 mimic control, RNAiAKT1 and RNAi-control vectors, respectively, were measured using the 3-(4,5-dimethylthiazol-2- yl)-2,5diphenyltetrazolium bromide (MTT) method following the protocol of our previous studies [13,50]. All experiments were done in triplicate. Mean normalized gene expression \pm SE was calculated from independent experiments.

\section{In vitro invasion and migration assay}

Cell invasion and migration were respectively analyzed by Matrigel coated transwell cell culture chambers $(8 \mu \mathrm{m}$ pore size, Millipore, Billerica, MA, USA) and the scratch wound-healing motility assay following the protocol of our previous studies $[13,50]$. All experiments were done in triplicate. Mean normalized gene expression \pm SE was calculated from independent experiments.

\section{Statistical analysis}

The software of SPSS version13.0 for Windows (SPSS Inc, IL, USA) and SAS 9.1 (SAS Institute, Cary, NC) was used for statistical analysis. The chi-squared test was used to show differences in categorical variables. Patient survival and the differences in patient survival were determined by the Kaplan-Meier method and the log-rank test, respectively. A Cox regression analysis (proportional hazard model) was performed for the multivariate analyses of prognostic factors. Differences were considered statistically significant when $\mathrm{P}$ was less than 0.05 .

\section{Additional files}

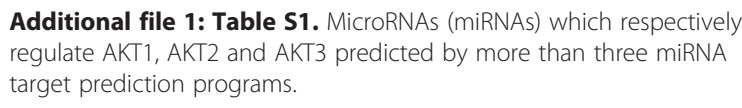

Additional file 3: Table S3. Validated targets for six candidate miRNAs collected from miRTarBase.

Additional file 4: Figure S1. Enriched gene ontology (GO) biological processes (A) and KEGG pathways (B) involved by involved by validated target genes of hsa-miR-149, hsa-miR-302d, hsa-miR-184, hsa-miR-708, hsa-miR-122 and hsa-miR-124. ${ }^{\prime * \prime} \mathrm{P}<0.01$; ${ }^{\prime * * \prime} \mathrm{P}<0.001$.

Additional file 5: Table S4. Interaction information of proteins encoded by validated targets of 6 candidate miRNAs.

Additional file 6: Table S5. Interaction information of hub nodes in the candidate miRNAs-regulated protein interaction network.

Additional file 7: Table S6. Topological features of 28 important nodes screened from miRNAs-regulated protein interaction network.

Additional file 8: Table S7. Edge betweenness for each interaction in the interaction network of hub proteins.

Additional file 9: Table S8. Detailed information on eight existing protein-protein interaction databases.

Additional file 10: Table S9. MicroRNAs (miRNAs) and mRNAs detected by qRT-PCR and their primers.

\section{Competing interests}

The authors declare that they have no competing interests.

\section{Authors' contributions}

LN and LB participated in study design and coordination, material support for obtained funding, and supervised study. ZY and GXD: performed the data analysis, designed the experimental validation and drafted the manuscript. XL, YLX, LZW, GQY and LZY: carried out the experimental validation. All authors read and approved the final manuscript.

\section{Acknowledgements}

National Natural Science Foundation of China (grant number. 81303153), State Project for Essential Drug Research and Development (grant number 
2013ZX09301307), National Basic Research Program of China (973 Program) (grant numbers 2011CB505300, 2011CB505305), \& Beijing Joint Project Specific Funds and the Fundamental Research Funds for the Central public welfare research institutes (Nos. ZZ2014035, ZZ2014036).

\section{Author details}

${ }^{1}$ Institute of Chinese Materia Medica, China Academy of Chinese Medical Sciences, Beijing 100700, China. ${ }^{2} 302$ Hospital of PLA, Beijing 100039, China. ${ }^{3}$ Beijing Zhongguancun Hospital, Beijing 100190, China.

Received: 24 August 2014 Accepted: 19 November 2014 Published: 26 November 2014

\section{References}

1. Jemal A, Bray F, Center MM, Ferlay J, Ward E, Forman D: Global cancer statistics. CA Cancer J Clin 2011, 61:69-90.

2. Forner A, Llovet JM, Bruix J: Hepatocellular carcinoma. Lancet 2012, 379:1245-1255.

3. Sherman M: Recurrence of hepatocellular carcinoma. N Engl J Med 2008, 359:2045-2047.

4. Thorgeirsson SS, Grisham JW: Molecular pathogenesis of human hepatocellular carcinoma. Nat Genet 2002, 31:339-346.

5. Bartel DP: MicroRNAs: genomics, biogenesis, mechanism, and function. Cell 2004, 116:281-297.

6. Han ZB, Zhong L, Teng MJ, Fan JW, Tang HM, Wu JY, Chen HY, Wang ZW, Qiu GQ, Peng ZH: Identification of recurrence-related microRNAs in hepatocellular carcinoma following liver transplantation. Mol Oncol 2012, 6:445-457.

7. Esquela-Kerscher A, Slack FJ: Oncomirs: microRNAs with a role in cancer. Nat Rev Cancer 2006, 6:259-269.

8. Wang J, Li J, Shen J, Wang C, Yang L, Zhang X: MicroRNA-182 downregulates metastasis suppressor 1 and contributes to metastasis of hepatocellular carcinoma. BMC Cancer 2012, 12:227.

9. Xue J, Niu J, Wu J, Wu ZH: MicroRNAs in cancer therapeutic response: Friend and foe. World J Clin Oncol 2014, 5:730-743.

10. Huang YH, Lin KH, Chen HC, Chang ML, Hsu CW, Lai MW, Chen TC, Lee WC, Tseng $\mathrm{YH}$, Yeh $\mathrm{CT}$ : Identification of postoperative prognostic microRNA predictors in hepatocellular carcinoma. PLoS One 2012, 7:e37188.

11. Shen G, Rong X, Zhao J, Yang X, Li H, Jiang H, Zhou Q, Ji T, Huang S, Zhang J, Jia H: MicroRNA-105 suppresses cell proliferation and inhibits $\mathrm{PI3K} / \mathrm{AKT}$ signaling in human hepatocellular carcinoma. Carcinogenesis 2014. In press.

12. Tan Y, Ge G, Pan T, Wen D, Chen L, Yu X, Zhou X, Gan J: A Serum MicroRNA Panel as Potential Biomarkers for Hepatocellular Carcinoma Related with Hepatitis B Virus. PLOS One 2014, 9:e107986.

13. Zhang Y, Guo X, Xiong L, Kong X, Xu Y, Liu C, Zou L, Li Z, Zhao J, Lin N: MicroRNA-101 suppresses SOX9-dependent tumorigenicity and promotes favorable prognosis of human hepatocellular carcinoma. FEBS Lett 2012, 586:4362-4370.

14. Huang JT, Wang J, Srivastava V, Sen S, Liu SM: MicroRNA Machinery Genes as Novel Biomarkers for Cancer. Front Oncol 2014, 4:113.

15. Zhang Y, Guo X, Yang M, Yu L, Li Z, Lin N: Identification of AKT kinases as unfavorable prognostic factors for hepatocellular carcinoma by a combination of expression profile, interaction network analysis and clinical validation. Mol Biosyst 2014, 10:215-222.

16. Li S, Zhang ZQ, Wu LJ, Zhang XG, Li YD, Wang YY: Understanding ZHENG in traditional Chinese medicine in the context of neuro-endocrineimmune network. IET Syst Biol 2007, 1:51-60.

17. Zhang Y, Li Z, Yang M, Wang D, Yu L, Guo C, Guo X, Lin N: Identification of GRB2 and GAB1 coexpression as an unfavorable prognostic factor for hepatocellular carcinoma by a combination of expression profile and network analysis. PLoS One 2013, 8:e85170.

18. Pan SJ, Zhan SK, Pei BG, Sun QF, Bian LG, Sun BM: MicroRNA-149 inhibits proliferation and invasion of glioma cells via blockade of AKT1 signaling. Int J Immunopathol Pharmacol 2012, 25:871-881.

19. Lin RJ, Lin YC: Yu AL: miR-149* induces apoptosis by inhibiting Akt1 and E2F1 in human cancer cells. Mol Carcinog 2010, 49:719-727.

20. Yu H, Kim PM, Sprecher E, Trifonov V, Gerstein M: The importance of bottlenecks in protein networks: Correlation with gene essentiality and expression dynamics. PLoS Comput Biol 2007, 3:e59.
21. Mohamed JS, Hajira A, Pardo PS, Boriek AM: MicroRNA-149 Inhibits PARP-2 and Promotes Mitochondrial Biogenesis via SIRT-1/PGC-1a Network in Skeletal Muscle. Diabetes 2014, 63:1546-1559.

22. Chan SH, Huang WC, Chang JW, Chang KJ, Kuo WH, Wang MY, Lin KY, Uen YH, Hou MF, Lin CM, Jang TH, Tu CW, Lee YR, Lee YH, Tien MT, Wang LH: MicroRNA-149 targets GIT1 to suppress integrin signaling and breast cancer metastasis. Oncogene 2014, 33:4496-4507.

23. Cheng T, Wang L, Li Y, Huang C, Zeng L, Yang J: Differential microRNA expression in renal cell carcinoma. Oncol Lett 2013, 6:769-776.

24. Ke Y, Zhao W, Xiong J: Cao R: miR-149 Inhibits Non-Small-Cell Lung Cancer Cells EMT by Targeting FOXM1. Biochem Res Int 2013, 2013:506731.

25. Luo Z, Zhang L, Li Z, Li X, Li G, Yu H, Jiang C, Dai Y, Guo X, Xiang J, Li G: An in silico analysis of dynamic changes in microRNA expression profiles in stepwise development of nasopharyngeal carcinoma. BMC Med Genet 2012, 19:3-14

26. Jin L, Hu WL, Jiang CC, Wang JX, Han CC, Chu P, Zhang $\sqcup$, Thorne RF, Wilmott J, Scolyer RA, Hersey P, Zhang XD, Wu M: MicroRNA-149*, a p53-responsive microRNA, functions as an oncogenic regulator in human melanoma. Proc Natl Acad Sci 2011, 108:15840-15845.

27. Chen YL, Uen YH, Li CF, Horng KC, Chen LR, Wu WR, Tseng HY, Huang HY, Wu LC, Shiue YL: The E2F transcription factor 1 transactives stathmin 1 in hepatocellular carcinoma. Ann Surg Oncol 2013, 20:4041-4054.

28. Frau M, Ladu S, Calvisi DF, Simile MM, Bonelli P, Daino L, Tomasi ML, Seddaiu MA, Feo F, Pascale RM: Mybl2 expression is under genetic control and contributes to determine a hepatocellular carcinoma susceptible phenotype. J Hepatol 2011, 55:111-119.

29. Nakajima T, Yasui K, Zen K, Inagaki Y, Fujii H, Minami M, Tanaka S, Taniwaki M, Itoh Y, Arii S, Inazawa J, Okanoue T: Activation of B-Myb by E2F1 in hepatocellular carcinoma. Hepatol Res 2008, 38:886-895.

30. Wang Y, Zheng X, Zhang Z, Zhou J, Zhao G, Yang J, Xia L, Wang R, Cai X, Hu H, Zhu C, Nie Y, Wu K, Zhang D, Fan D: MicroRNA-149 Inhibits Proliferation and Cell Cycle Progression through the Targeting of ZBTB2 in Human Gastric Cancer. PLoS One 2012, 7:e41693.

31. Lewis BP, Burge CB, Bartel DP: Conserved seed pairing, often flanked by adenosines, indicates that thousands of human genes are microRNA targets. Cell 2005, 120:15-20.

32. Wang $X$ : MiRDB: a microRNA target prediction and functional annotation database with a wiki interface. RNA 2008, 14:1012-1017.

33. Kiriakidou M, Nelson PT, Kouranov A, Fitziev P, Bouyioukos C, Mourelatos Z: A combined computational-experimental approach predicts human microRNA targets. Genes Dev 2004, 18:1165-1178.

34. John B, Enright AJ, Aravin A, Tuschl T, Sander C, Marks DS: Human microRNA targets. PLoS Biol 2004, 2:e363.

35. Krek A, Grun D, Poy MN, Wolf R, Rosenberg L, Epstein EJ: Combinatorial microRNA target predictions. Nat Genet 2005, 37:495-500.

36. Rehmsmeier M, Steffen $P$, Hochsmann M, Giegerich R: Fast and effective prediction of microRNA/target duplexes. RNA 2004, 10:1507-1517.

37. Hsu SD, Tseng YT, Shrestha S, Lin YL, Khaleel A, Chou CH, Chu CF, Huang HY, Lin CM, Ho SY, Jian TY, Lin FM, Chang TH, Weng SL, Liao KW, Liao IE, Liu CC: Huang HD: miRTarBase update 2014: an information resource for experimentally validated miRNA-target interactions. Nucleic Acids Res 2014, 42(Database issue):D78-D85.

38. Dennis G Jr, Sherman BT, Hosack DA, Yang J, Gao W: DAVID: Database for Annotation, Visualization, and Integrated Discovery. Genome Biol 2003, 4:P3.

39. Wixon J, Kell D: The Kyoto encyclopedia of genes and genomes-KEGG. Yeast 2000, 17:48-55.

40. Chen JY, Mamidipalli S, Huan T: HAPPI: an online database of comprehensive human annotated and predicted protein interactions. BMC Genomics 2009, 10(Suppl 1):S16.

41. Matthews L, Gopinath G, Gillespie M, Caudy M, Croft D: Reactome knowledgebase of human biological pathways and processes. Nucleic Acids Res 2009, 37:D619-D622.

42. Brown KR, Jurisica I: Online predicted human interaction database. Bioinformatics 2005, 21:2076-2082.

43. Aranda B, Achuthan P, Alam-Faruque Y, Armean I, Bridge A: The IntAct molecular interaction database in 2010. Nucleic Acids Res 2010, 38:D525-D531.

44. Keshava Prasad TS, Goel R, Kandasamy K, Keerthikumar S, Kumar S: Human Protein Reference Database - 2009 update. Nucleic Acids Res 2009, 37:D767-D772. 
45. Ceol A, Chatr Aryamontri A, Licata L, Peluso D, Briganti L: MINT, the molecular interaction database: 2009 update. Nucleic Acids Res 2010, 38:D532-D539.

46. Lehne B, Schlitt T: Protein-protein interaction databases: keeping up with growing interactomes. Hum Genomics 2009, 3:291-297.

47. Beuming T, Skrabanek L, Niv MY, Mukherjee P, Weinstein H: PDZBase: a protein-protein interaction database for PDZ-domains. Bioinformatics 2005, 21:827-828.

48. Valente TW, Fujimoto K: Bridging: locating critical connectors in a network. Soc Networks 2010, 32:212-220.

49. Narayanan T, Gersten M, Subramaniam S, Grama A: Modularity detection in protein-protein interaction networks. BMC Res Notes 2011, 4:569.

50. Lin ZY, Huang YQ, Zhang YQ, Han ZD, He HC, Ling XH, Fu X, Dai QS, Cai C, Chen JH, Liang YX, Jiang FN, Zhong WD, Wang F, Wu CL: MicroRNA-224 inhibits progression of human prostate cancer by downregulating TRIB1. Int J Cancer 2014, 135:541-550.

doi:10.1186/1476-4598-13-253

Cite this article as: Zhang et al:: Comprehensive analysis of microRNAregulated protein interaction network reveals the tumor suppressive role of microRNA-149 in human hepatocellular carcinoma via targeting AKT-mTOR pathway. Molecular Cancer 2014 13:253.

\section{Submit your next manuscript to BioMed Central and take full advantage of:}

- Convenient online submission

- Thorough peer review

- No space constraints or color figure charges

- Immediate publication on acceptance

- Inclusion in PubMed, CAS, Scopus and Google Scholar

- Research which is freely available for redistribution 